\title{
Exciton Polaritons Confined in a ZnO Nanowire Cavity
}

\author{
Lambert K. van Vugt, ${ }^{1}$ Sven Rühle, ${ }^{1}$ Prasanth Ravindran, ${ }^{2}$ Hans C. Gerritsen, ${ }^{2}$ \\ Laurens Kuipers, ${ }^{3}$ and Daniël Vanmaekelbergh ${ }^{1}$ \\ ${ }^{1}$ Condensed Matter and Interfaces, Debye Institute, Utrecht University, Post Office Box 80000, 3508 TA Utrecht, The Netherlands \\ ${ }^{2}$ Molecular Biophysics, Debye Institute, Utrecht University, Post Office Box 80000, 3508 TA Utrecht, The Netherlands \\ ${ }^{3}$ Center for Nanophotonics, FOM Institute for Atomic and Molecular Physics (AMOLF),
}

Kruislaan 407, 1098 SJ Amsterdam, The Netherlands

(Received 10 May 2006; published 5 October 2006)

\begin{abstract}
Semiconductor nanowires of high purity and crystallinity hold promise as building blocks for miniaturized optoelectrical devices. Using scanning-excitation single-wire emission spectroscopy, with either a laser or an electron beam as a spatially resolved excitation source, we observe standing-wave exciton polaritons in $\mathrm{ZnO}$ nanowires at room temperature. The Rabi splitting between the polariton branches is more than $100 \mathrm{meV}$. The dispersion curve of the modes in the nanowire is substantially modified due to light-matter interaction. This finding forms a key aspect in understanding subwavelength guiding in these nanowires.
\end{abstract}

DOI: 10.1103/PhysRevLett.97.147401

Chemically prepared semiconductor nanowires form a class of very promising building blocks for miniaturized optical and electrical devices [1]. They possess a high degree of crystallinity and the lattice orientation is often well defined with respect to the nanowire geometry [2]. Semiconductor nanowires offer the possibility of photonexciton conversion and interaction: a key aspect in quantum optics and optoelectrical applications. For instance, light-emitting diodes based on crossed $p$-type and $n$-type nanowires [3] and wire-integrated $p$ - $n$ junctions have been reported [4], as well as nanowire lasers [5] and fieldselective photon detectors [6]. It is well known that optical properties can be affected in resonant structures. In semiconductor microcavities, strong exciton-photon coupling, leading to exciton polaritons with an avoided crossing of the photon and exciton dispersion curves, has been reported [7-9]. The normal-mode cavity splitting, in this field commonly referred to as the Rabi splitting, was at most $10 \mathrm{meV}$.

Crystalline $\mathrm{ZnO}$ forms an intriguing system with regard to the interaction between excitons and photons. There are three excitonic transitions, between 3.30 and $3.36 \mathrm{eV}$. The electron-hole binding energy is about $60 \mathrm{meV}$ [10]; excitons are thus stable at room temperature. Furthermore, the exciton transitions have a large oscillator strength, which should favor light-matter interaction. Exciton-photon coupling has been shown to exist and affect the light dispersion relation in macroscopic bulk $\mathrm{ZnO}$ [11]. This has led to a strong interest in the optical properties of $\mathrm{ZnO}$ up to date. Reflectivity measurements on macroscopic $\mathrm{ZnO}$ revealed a very large longitudinal-transverse splitting of about $5 \mathrm{meV}$, 2 orders of magnitude larger than in GaAs [12]. On thin films of $\mathrm{ZnO}$, exciton polaritons have been studied by their photoluminescence spectrum up to $250 \mathrm{~K}$ [13]. It was anticipated that strong light-matter interaction should prevail in $\mathrm{ZnO}$ photonic nanostructures due to a combination of photon confinement and strong exciton absorption [14,15].
PACS numbers: 78.67.Pt, 71.36.+c, 71.55.Gs, 78.66.Hf

In this Letter we report extremely strong exciton-photon coupling in $\mathrm{ZnO}$ nanowires at room temperature. We collect emission spectra of single wires upon scanning a focused laser or electron excitation spot along the wire. We report strongly enhanced excitation probabilities at the wire ends for two groups of polariton modes, separated by a gap of 60-164 meV. Our results thus provide strong evidence for a giant Rabi splitting. This strong excitonphoton interaction determines the polaritonic dispersion curve in the near UV and might explain the surprising subwavelength guiding that has been reported previously and which is also observed with the wires used in this study.

In order to unravel the modelike properties of the excitation, single wires with known geometry have to be investigated. We therefore performed two-photon excitation luminescence spectroscopy and transmission electron microscopy (TEM) on the same individual $\mathrm{ZnO}$ nanowires. $\mathrm{ZnO}$ wires of length between 1 and $10 \mu \mathrm{m}$ along the $c$ axis and diameter between 100 and $300 \mathrm{~nm}$ were investigated. The $\mathrm{ZnO}$ nanowires were grown on a sapphire substrate with the vapor-liquid-solid method at $920^{\circ} \mathrm{C}$ using gold as a catalyst $[16,17]$. After growth, the wires were dispersed on a $20 \mathrm{~nm}$ thick silicon nitride membrane, which allowed both optical and TEM characterization. The wires were excited with a Ti:sapphire $100 \mathrm{fs}$ pulsed laser with variable photon energy $(1.36 \mathrm{eV}<\hbar \omega<1.77 \mathrm{eV})$. The $\mathrm{ZnO}$ wires show strong emission at doubled frequency, i.e., second harmonic ( $\mathrm{SH})$ generation [18]. When $2 \hbar \omega$ is below the typical exciton transition energy in a $\mathrm{ZnO}$ crystal, around $3.31 \mathrm{eV}$, only SH emission is observed. However, when $2 \hbar \omega$ is resonant with or higher in energy than the exciton transitions, the wire also luminesces in the UV and the green. A typical luminescence spectrum of a single crystal $\mathrm{ZnO}$ nanowire excited with $2 \hbar \omega=3.44 \mathrm{eV}$, i.e., above the exciton transitions, is shown in Fig. 1(a). The emitted light of doubled frequency $(\mathrm{SH})$ is clearly visible as a small peak 
at $3.44 \mathrm{eV}$. In addition, there is a strong luminescence peak at around $3.26 \mathrm{eV}$, which, for bulk $\mathrm{ZnO}$, has been ascribed to emission from the lower polariton branch [13]. The broad green luminescence peak $\left(\hbar \omega_{e}<2.8 \mathrm{eV}\right)$ arises from trapping of the excitons in a defect center [19]. The SH and UV emissions are coupled: their intensity depends strongly and in the same way on the polarization of the excitation beam with respect to the $c$ axis (long axis) of the $\mathrm{ZnO}$ wire.

In macroscopic crystals exciton polaritons are conventionally studied with angle-dependent reflection spectroscopy. This is not possible with single wires of subwavelength diameter. Here, we collect the emitted spectra of a single nanowire upon scanning with a spatially resolved excitation spot (400 $\mathrm{nm}$ spatial resolution) along the wire. The total single-wire emission spectrum was measured for each position of the excitation spot along the wire. The spatial pattern of the single-wire emission at given emission energy $\hbar \omega_{e}$ thus represents the spatially resolved probability of exciting modes of energy $\hbar \omega_{e} \pm 5 \mathrm{meV}(10 \mathrm{meV}$ bandwidth of the detection system) in a given $\mathrm{ZnO}$ nanowire [20]. Such excitation patterns, for $2 \hbar \omega$ excitation above the exciton energy and selected emission energies, are presented in Figs. 1(b)-1(g). Five other wires that were investigated show qualitatively similar results. Surprisingly, we observe a strong enhancement of the excitation rate of UV emission $(3.25-3.40 \mathrm{eV})$ at the ends of the wire with respect to the center. The enhancement is also found for light emission at lower energy $(2.7-3.2 \mathrm{eV})$ on the condition that the two-photon excitation is resonant with or has a higher energy than the exciton transition.

Trivial causes or experimental artifacts, such as a more favorable incoupling of the excitation beam at the wire ends, can be excluded for a number of reasons. First, if the two-photon excitation $2 \hbar \omega$ is below the exciton transitions, only SH emission is observed, with a single-wire emission intensity that is independent of the position of the excitation spot. Incoupling effects at the wire ends would have resulted in enhancement of the $\mathrm{SH}$ as well, which is not observed. A nearly flat excitation pattern is also observed for the $\mathrm{SH}$ emission at $3.44 \mathrm{eV}$, i.e., above exciton transitions [Fig. 1(g), red line]. Second, for excitation with two-photon energy in resonance with or above the exciton transitions, we found that the excitation enhancement at the wire ends vanishes at high excitation intensity (see below). Third, exciton luminescence in $\mathrm{ZnO}$ nanowires can be generated by excitation with an electron beam rather than a laser focus [21]. Since an electron beam offers an excellent spatial resolution $(<50 \mathrm{~nm})$, we also measured the spatial excitation patterns of single-wire cathodoluminescence, which are very similar to the observed ones with two-photon excitation (not shown). In the exciton region, nonuniform excitation patterns are observed with strongly enhanced excitation rates at both wire ends. The spatial excitation patterns nearly vanish for emission modes below the exciton transitions. Hence, the spatial excitation patterns observed with electron-beam excitation depend in a similar way on the emission photon energy $\hbar \omega_{e}$ as those observed by two-photon excitation. It is clear that these observations, with photons or electrons as excitation source, exclude experimental artifacts as the cause of the observed excitation patterns and reflect the same underlying physics. Exciton formation is a prerequisite for the spatial patterns observed in the excitation rate along a $\mathrm{ZnO}$ nanowire. They must, therefore, result from a strong coupling of optical cavity modes with the exciton dipoles, i.e., exciton-polariton generation.

The enhanced excitation rate that we observe at the ends of the $\mathrm{ZnO}$ nanowires with respect to the center part can be understood by considering that, at a given emission energy $\hbar \omega_{e}$, the single-wire emission intensity is determined by the local generation probability of all modes within the bandwidth of the detection system $\Delta \omega(10 \mathrm{meV})$. The number of modes and their wave vector at each $\hbar \omega_{e} \pm$ $5 \mathrm{meV}$ are determined by the polariton dispersion relation in a given wire (see below). The modes at different energy
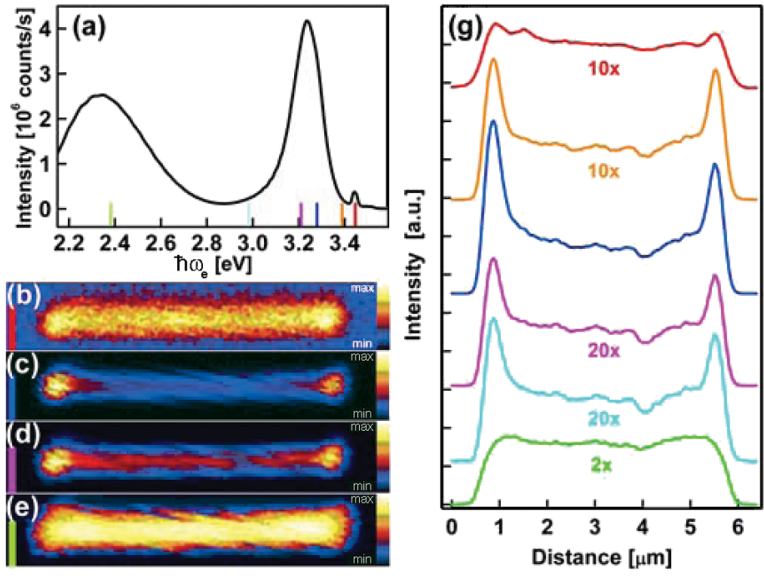

(f)

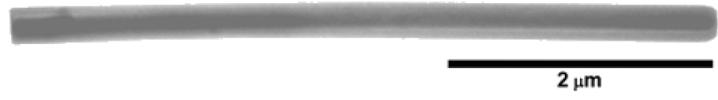

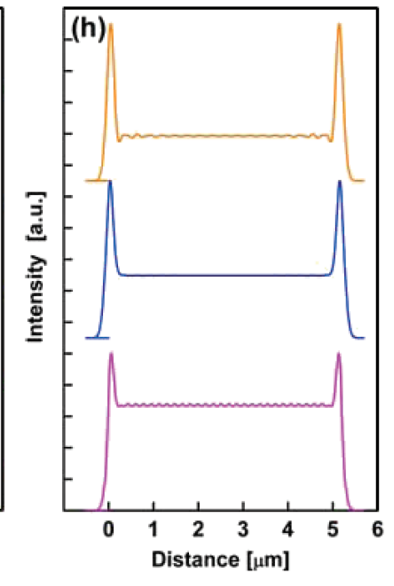

FIG. 1 (color). (a) Average photoluminescence spectrum of a $\mathrm{ZnO}$ nanowire for excitation with sub-band-gap light $(1.72 \mathrm{eV})$, showing green and UV photoluminescence as well as second harmonic generation. (b)-(e) Spatially resolved excitation images taken at the emission energies indicated in panel (a) by the colored bars. (f) TEM image of the investigated wire. The wire had a length of $5.19 \mu \mathrm{m}$ and a diameter of $273 \mathrm{~nm}$. (g) Excitation line traces along the length of the wire ( $c$ axis) at emission energies indicated by the colored bars in panel (a). The profiles are scaled with the indicated factors for clarity. (h) Calculated excitation profiles for the energies shown by the color bars in (a). 
interfere, which leads to temporal oscillations in the intensity decaying with a time constant $2 \pi / \Delta \omega$. Since all relevant time scales in the experiment $(\mathrm{CCD}$ response and integration time, repetition rate of the laser) are much longer than $2 \pi / \Delta \omega$, the detected emission is proportional to the number of modes excited at the excitation spot. Calculated excitation profiles along the wire for three different energies $\hbar \omega_{e}$ in the exciton region are presented in Fig. 1(h); details are given below.

To further investigate the dispersion properties of the polariton modes in a given nanowire, we studied the spatial excitation patterns as a function of the mode energy $\hbar \omega_{e}$ of the emitted light. We quantified the enhancement of the mode generation rate at each wire end by the enhancement factor $\mathrm{EF}\left(\hbar \omega_{e}\right)$, defined as the single-wire emission intensity at $\hbar \omega_{e}$ for excitation at a wire end divided by the single-wire emission for excitation at the center (spatially averaged over a few $\mu \mathrm{m})$. Spectra of $\operatorname{EF}\left(\hbar \omega_{e}\right)$ measured with two different wires are presented in Figs. 2(a) and 2(b), together with the emission spectra of the wires (averaged for excitation over the entire wire). The enhancement spectra of these two and other wires are very similar. For the discussion we focus on the spectrum shown in Fig. 2(b). Below a certain cutoff energy (at $2.71 \mathrm{eV}$ ), $\mathrm{EF}\left(\hbar \omega_{e}\right)$ is unity. There is a broad enhancement peak in the transparent region between the cutoff energy and $3.2 \mathrm{eV}$, with reproducible modulations due to Fabry-Pérot interference in the $\mathrm{ZnO}$ nanowire cavity. From the width of the interference peaks we determine a $Q$ factor of 256; note that this value holds only for the optical part between 2.7 and $3.0 \mathrm{eV}$. The most prominent features of the enhancement spectrum $\operatorname{EF}\left(\hbar \omega_{e}\right)$ are the two peaks in the exciton energy region between 3.2 and $3.5 \mathrm{eV}$. The low-energy peak at around $3.28 \mathrm{eV}$ coincides with the high-energy side of the exciton luminescence peak. Therefore, the low-energy peak is composed of a number of transverse modes; $\mathbf{E} \perp \mathbf{k} \|$ crystal $c$ axis, where $\mathbf{E}$ is the electric field vector and $\mathbf{k}$ the polariton wave vector. The second peak in the enhancement spectrum is centered at $3.38 \mathrm{eV}$; note that the exciton luminescence intensity is very low at this energy. We calculated the enhancement spectrum $\mathrm{EF}\left(\hbar \omega_{e}\right)$ from the exciton-polariton dispersion curve for a $\mathrm{ZnO}$ nanowire with the same length as in Fig. 2(b); we took into account lateral photon confinement and the dispersion relation given by [12]

$\varepsilon(\omega, k)=\varepsilon_{\infty}\left(1+\sum_{j=A, B, C} \Omega_{j} \frac{\omega_{j, L}^{2}-\omega_{j, T}^{2}}{\omega_{j, T}^{2}-\omega^{2}-i \omega \gamma_{j}}\right)=\frac{c^{2} k^{2}}{\omega^{2}}$,

with the background dielectric constant $\varepsilon_{\infty}$, the transverse $\left(\omega_{j, T}\right)$ and longitudinal $\left(\omega_{j, L}\right)$ resonance frequencies, the damping constants $\gamma_{j}$, the speed of light $c$ and a prefactor $\Omega_{j}$ as defined in Ref. [12]. The damping and the resonant frequencies (for $A, B$, and $C$ excitons) were taken as for a
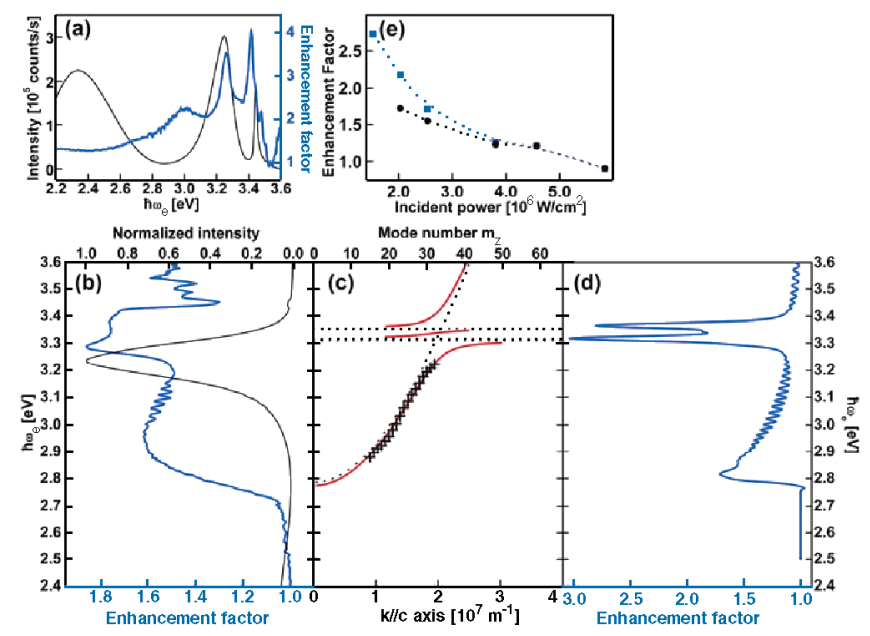

FIG. 2 (color). (a) Average spectrum (black line) and enhancement spectrum (blue line) of a wire excited with $\hbar \omega=1.72 \mathrm{eV}$, which shows, near the exciton resonances, a peak splitting in the enhancement spectrum of $164 \mathrm{meV}$. (b) Average spectrum (black line) and enhancement spectrum (blue line) of a wire excited with $\hbar \omega=1.72 \mathrm{eV}$. The enhancement spectrum shows a peak splitting of $102 \mathrm{meV}$ near the exciton resonances and modulations due to Fabry-Pérot interference in the transparent region (2.7-3.2 eV). (c) Exciton-polariton dispersion (red lines) calculated with Eq. (1) from the resonances of the $A(3.309 \mathrm{eV}), B$ $(3.315 \mathrm{eV})$, and $C(3.355 \mathrm{eV})$ excitons (dotted horizontal lines) and a photon mode confined in the nanowire cavity (dotted curve). For clarity, we do not show the strongly damped modes in the anticrossing energy-gap. The crosses indicate the energetic peak positions obtained from the Fabry-Pérot maxima of the enhancement spectrum shown in panel (b). These maxima are equidistantly spaced at $m_{z}{ }^{*}(\pi / L)$ in $k$ space $(L=$ the length of the wire $=5.19 \mu \mathrm{m}$ ) and give the slope of the exciton-polariton dispersion between 2.7 and $3.2 \mathrm{eV}$. (d) Calculated enhancement resulting from the dispersion curve shown in (c). (e) Excitation power dependence of the peaks in the enhancement spectrum.

macroscopic $\mathrm{ZnO}$ crystal [12] with the resonance frequencies shifted to room temperature. The calculated dispersion relation (without strongly damped modes) is shown in Fig. 2(c). From this dispersion relation we have calculated the excitation profiles between 2.5 and $3.5 \mathrm{eV}$ [see, for instance, Fig. 1(h)] and the ensuing enhancement spectrum [Fig. 2(d)]. We considered the modes present in each energy window $\hbar \omega_{e} \pm 5 \mathrm{meV}$ according to the polariton dispersion curve, convoluted the field of each standing wave with the laser profile, calculated the intensity, made the summation, and added a background to account for all excitations which are not proper polaritons. We made the reasonable assumption that this background is independent of the position along the wire and the energy. From these excitation profiles along the wire, we obtained $\operatorname{EF}\left(\hbar \omega_{e}\right)$; the resulting spectrum is shown in Fig. 2(d) [22]. The enhancement spectrum turns out to be sensitive to the slope of the dispersion curve; in regions with a small slope, more 
modes need to be included in the summation which leads to a higher enhancement factor. The essential features of the experimental enhancement spectrum (i.e., broad enhancement peak with Fabry-Pérot oscillations in the blue region) and the two peaks in the exciton region are well reproduced by the calculation. The enhancement in the energy range between the cutoff energy and $3.2 \mathrm{eV}$ is due to standingwave polariton modes with a strong photon character. The oscillations reflect the detection of single modes in the energy interval $\hbar \omega_{e} \pm 5 \mathrm{meV}$. The two peaks in the exciton region are due to a high energy density of the confined polariton modes, i.e., flat parts of the dispersion. Further calculations show that the peak separation increases with increasing oscillator strength (not shown). We propose that the measured gap between both groups of polariton modes (between 60 and $164 \mathrm{meV}$ ) is hence the result of increased exciton-photon coupling in the wires. We remark that the enhancement of the excitation at the wire ends decreases with increasing excitation intensity [see Fig. 2(e)]. This can be explained by dissociation of the excitons and hence weakening of the light-matter interaction. This might be due to increased screening, exciton-exciton scattering, or even increased temperature of the wire.

The energy difference between the two groups of polariton modes forms a lower limit for the Rabi splitting, a measure of the strength of the exciton-photon coupling. In Fig. 2(b) we observe a splitting of about $100 \mathrm{meV}$. Several other wires have shown an even larger splitting up to $164 \mathrm{meV}$ [see Fig. 2(a)]. We note that the energy separation between the two groups of polariton modes is considerably larger than the energy difference between the $A, B$, and $C$ excitons in bulk $\mathrm{ZnO}$ of about $50 \mathrm{meV}$ [10]. Our results indicate strong light-matter interaction in $\mathrm{ZnO}$ nanowire cavities. This must be due to the large oscillator strength of the exciton transitions in $\mathrm{ZnO}$ and the small mode volume $[14,15,23]$. Recently, it was reported that GaN microcavities show two resonance peaks in the reflection spectra, with a Rabi splitting of about $50 \mathrm{meV}$ [24]. We remark that an even larger Rabi splitting has been observed in an organic system [25]. A Rabi splitting of $120 \mathrm{meV}$ was predicted for a $\mathrm{ZnO} \lambda$ cavity with distributed Bragg mirrors [15]. Our results provide the first strong evidence that such a large splitting can be reached, even in a simple nanowire geometry. Theoretical efforts are needed for a detailed and more quantitative explanation.

Since both the electron-hole binding energy $(60 \mathrm{meV})$ and the Rabi splitting are considerable larger than $\mathrm{kT}$, exciton-photon coupling plays an important role in the optical properties of $\mathrm{ZnO}$ nanowires at room temperature. The strongly modified light dispersion curve [Fig. 2(c)] should be taken into account if $\mathrm{ZnO}$ nanowires are applied in photonic circuits. Impressive subwavelength guiding has been reported in $\mathrm{ZnO}$ nanowires [26]; we observed similar guiding in our experiments. We propose that this guiding is mediated by the transverse exciton polaritons (lower branch at around $3.28 \mathrm{eV}$ ). The observed delocalization of polaritons with high wave numbers over the entire wire length is paramount to waveguiding with small losses. The possibility of generating polariton modes at room temperature, which strongly vary in their wave properties for small changes in energy, could be of huge importance for nanophotonic circuitry.

This work is part of the research program of the "Stichting voor Fundamenteel Onderzoek der Materie (FOM)," which is financially supported by the Nederlandse Organisatie voor Wetenschappelijk Onderzoek (NWO). This research is supported by NanoNed, a national nanotechnology program coordinated by the Dutch Ministry of Economic Affairs. This work was made possible by the fabrication and characterization facilities of the Amsterdam nanoCenter.

[1] Y. Xia et al., Adv. Mater. 15, 353 (2003).

[2] B. A. Wacaser et al., J. Cryst. Growth 287, 504 (2006).

[3] X. Duan et al., Nature (London) 409, 66 (2001).

[4] M.S. Gudiksen et al., Nature (London) 415, 617 (2002).

[5] M. H. Huang et al., Science 292, 1897 (2001).

[6] J. Wang et al., Science 293, 1455 (2001).

[7] C. Weisbuch et al., Phys. Rev. Lett. 69, 3314 (1992).

[8] J.P. Reithmaier et al., Nature (London) 432, 197 (2004).

[9] T. Yoshie et al., Nature (London) 432, 200 (2004).

[10] D. G. Thomas, J. Phys. Chem. Solids 15, 86 (1960).

[11] J. J. Hopfield and D. G. Thomas, Phys. Rev. Lett. 15, 22 (1965).

[12] J. Lagois, Phys. Rev. B 23, 5511 (1981).

[13] A. A. Toropov et al., Phys. Rev. B 69, 165205 (2004).

[14] B. Gil and A. V. Kavokin, Appl. Phys. Lett. 81, 748 (2002).

[15] M. Zamfirescu et al., Phys. Rev. B 65, 161205(R) (2002).

[16] M. H. Huang et al., Adv. Mater. 13, 113 (2001).

[17] R. Prasanth et al., Appl. Phys. Lett. 88, 181501 (2006).

[18] D. Bogget and R. Loudon, J. Phys. C 6, 1763 (1973).

[19] A. van Dijken et al., J. Lumin. 87-89, 454 (2000).

[20] The difference in excitation energy $2 \hbar \omega$ and emitted energy $\hbar \omega_{e}$ is lost in phonons. We assume that this loss does not depend on the position of excitation.

[21] H. J. Fan et al., Appl. Phys. Lett. 86, 023113 (2005).

[22] See EPAPS Document No. E-PRLTAO-97-027640 for a detailed calculation procedure of the enhancement spectrum. For more information on EPAPS, see http://www. aip.org/pubservs/epaps.html.

[23] M. Bayer et al., Phys. Status Solidi A 191, 3 (2002).

[24] I. R. Sellers et al., Phys. Rev. B 73, 033304 (2006).

[25] D. G. Lidzey et al., Nature (London) 395, 53 (1998).

[26] M. Law et al., Science 305, 1269 (2004). 